\title{
Fatal breathing dysfunction in a mouse model of Leigh syndrome
}

\author{
Albert Quintana, ${ }^{1}$ Sebastien Zanella, ${ }^{2}$ Henner Koch, ${ }^{2}$ Shane E. Kruse, ${ }^{1}$ \\ Donghoon Lee, ${ }^{3}$ Jan M. Ramirez, ${ }^{2}$ and Richard D. Palmiter ${ }^{1}$
}

\begin{abstract}
${ }^{1}$ Howard Hughes Medical Institute and Department of Biochemistry, ${ }^{2}$ Center for Integrative Brain Research, Seattle Children's Research Institute and
\end{abstract} Department of Neurological Surgery, and ${ }^{3}$ Department of Radiology, University of Washington, Seattle, Washington, USA.

\begin{abstract}
Leigh syndrome (LS) is a subacute necrotizing encephalomyelopathy with gliosis in several brain regions that usually results in infantile death. Loss of murine Ndufs 4 , which encodes NADH dehydrogenase (ubiquinone) iron-sulfur protein 4, results in compromised activity of mitochondrial complex I as well as progressive neurodegenerative and behavioral changes that resemble LS. Here, we report the development of breathing abnormalities in a murine model of LS. Magnetic resonance imaging revealed hyperintense bilateral lesions in the dorsal brain stem vestibular nucleus (VN) and cerebellum of severely affected mice. The mutant mice manifested a progressive increase in apnea and had aberrant responses to hypoxia. Electrophysiological recordings within the ventral brain stem pre-Bötzinger respiratory complex were also abnormal. Selective inactivation of $N d u f s 4$ in the $\mathrm{VN}$, one of the principle sites of gliosis, also led to breathing abnormalities and premature death. Conversely, $N d u f s 4$ restoration in the VN corrected breathing deficits and prolonged the life span of knockout mice. These data demonstrate that mitochondrial dysfunction within the VN results in aberrant regulation of respiration and contributes to the lethality of $N d u f s 4$-knockout mice.
\end{abstract}

\section{Introduction}

Leigh syndrome (LS), a progressive neurodegenerative disorder (1), is the most common infantile mitochondrial disorder, affecting 1 in 40,000 live births (2). MRI reveals bilateral hyperintense lesions in many brain regions of LS patients $(3,4)$ that correlate with gliosis, demyelination, capillary proliferation, and/or necrosis (5). Behavioral symptoms of LS patients vary widely, but can include developmental retardation, hypotonia, ataxia, dystonia, optic atrophy, hearing impairment, breathing abnormalities, dysarthria, swallowing difficulties, and failure to thrive $(3,6,7)$. Respiratory arrest, the most common cause of death for LS patients, is observed in up to $75 \%$ of cases $(8,9)$. The necessity for mechanical ventilation or the occurrence of sudden death of LS patients is associated with brain stem lesions $(3,10)$. However, case report data are usually scarce, and the mechanisms involved in the respiratory apnea in LS cases are unclear. The lack of a genetic model to study disease progression and the cause of death has impeded a rigorous investigation.

The most prevalent mitochondrial disorders affect complex I (11-13), and complex I deficiencies are detected in many LS patients $(4,14,15)$. Mutations in the nuclear gene encoding in NDUFS4 (NADH dehydrogenase [ubiquinone] iron-sulfur protein 4), one of the 45 subunits of complex I, have been described as causing LS in humans (16-20). Human LS patients harboring NDUFS4 mutations develop brain stem and basal ganglia lesions and die from cardiorespiratory failure at an early age, with marked apneic episodes in the majority of cases along with a minor presence of hypertrophic cardiomyopathy (21). We developed a line of mice in which the $N d u f s 4$ gene can be inactivated by Cre recombinase (22). Here, we delineate the cause of death of Ndufs4-deficient mice and develop a gene therapy strategy.

Conflict of interest: The authors have declared that no conflict of interest exists. Citation for this article: J Clin Invest. 2012;122(7):2359-2368. doi:10.1172/JCI62923.

\section{Results}

KO mice developsymptoms similarto those of humanswith NDUFS4 mutations. Mice with both alleles of the Ndufs 4 gene inactivated in all cells or just in the CNS manifest a fatal progressive encephalopathy and behavioral characteristics similar to those of people with LS, especially those with NDUFS4 mutations (21-23). The similarities include failure to thrive, growth retardation, ataxia, hypotonia, visual problems, and breathing irregularities (Table 1). Brain lesions detected by imaging and histology are also similar (24).

Brain lesions in KO mice are detected by MRI and gliosis. T2-weighted MRI reveals edematous regions as a hyperintense signal that is a primary diagnostic sign for LS. Of $5 \mathrm{KO}$ mice examined, 2 midstage and 2 late-stage mice (as defined in the methods section) had hyperintense lesions in the deep cerebellar fastigial nucleus (FN), cerebellar lobes (predominantly posterior lobes such as VIII, IX, X, but extending to anterior lobes in late-stage animals), the external plexiform layer of the olfactory bulb (OB), and the dorsal medulla in either sagittal or coronal views that are not seen in control mice (Figure 1A). Lesions in the dorsal medulla appear to be in the vestibular nucleus (VN) (Figure 1B). Gliosis develops progressively in these same brain regions, as revealed by immunostaining with antibodies that detect glial reactivity. For example, immunostaining for glial markers reveals extensive bilateral neuroinflammation in the $\mathrm{VN}$ of KO mice (Figure 1C). Neurons with cytoplasmic vacuoles and aberrant mitochondria containing condensed cristae as well as microglia engorged with cytoplasmic remnants were observed at the ultrastructural level in the same brain regions (23). The severe encephalopathy leads to a shortened life span with greater than $90 \%$ mortality by P50 (Figure 1D).

$\mathrm{KO}$ mice have abnormal respiratory responses. $\mathrm{KO}$ mice are small and manifest hypothermia, ataxia, lethargy, and sensory abnormalities $(22,23)$. The cerebellar and VN lesions could easily account for the ataxia, but the underlying cause of the other symptoms and death remained enigmatic until we observed intermittent breathing irregularities. KO mice lose most of their hair at approximate- 
Table 1

Clinical observations in LS and Ndufs4-KO mice

$\begin{array}{lccc}\text { Human LS } & \begin{array}{c}\text { NDUFS4-LS } \\ \text { patients } \\ \mathbf{( 2 1 )}\end{array} & \begin{array}{c}\text { Ndufs4-KO } \\ \text { mouse } \\ \mathbf{( 2 2 )}\end{array} & \begin{array}{c}\text { CNS-specific } \\ \text { Ndufs4-KO }\end{array} \\ \text { Abnormal brain images } & 15 / 15 & \text { Yes } & \text { Yes } \\ \text { Brain stem } & 11 / 12 & \text { Yes } & \text { Yes } \\ \text { Basal ganglia } & 9 / 12 & \text { No/Yes } & \text { No } \\ \text { Ataxia, motor alterations } & \text { Yes (18) } & \text { Yes } & \text { Yes } \\ \text { Growth retardation } & 6 / 12 & \text { Yes } & \text { Yes } \\ \text { Failure to thrive } & 7 / 7 & \text { Yes } & \text { Yes } \\ \text { Hypotonia } & 18 / 18 & \text { Yes } & \text { Yes } \\ \text { Visual problems } & 7 / 7 & \text { Yes } & \text { Yes } \\ \text { Breathing irregularities } & 10 / 12 & \text { Yes }^{\mathrm{A}} & \text { Yes } \\ \text { Apneic episodes } & 10 / 12 & \text { Yes }^{\mathrm{A}} & \text { Yes }^{\mathrm{A}} \\ & & & \end{array}$

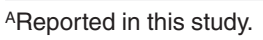

ly P20, which facilitates observing breathing irregularities that include hypo- or hyperventilation and gasping (Supplemental Video 1; supplemental material available online with this article; doi:10.1172/JCI62923DS1). The appearance of gasping-like episodes was commonly observed under more stressful conditions, for example, when mice were handled.

To delineate respiratory responses of $\mathrm{KO}$ mice, we used barometric, whole-body, flow plethysmography to record the breathing of unanesthetized, freely moving $\mathrm{KO}$ and control mice (Figure 2A). Under normoxic conditions $\left(20.9 \% \mathrm{O}_{2}\right)$, neonate (P8-P12) and older $(>\mathrm{P} 30) \mathrm{KO}$ mice had normal respiratory frequency ( $f R$ ); however, they had significantly higher tidal volume $\left(V_{T}\right)$ and minute ventilation $\left(V_{E}=f R \times V_{T}\right)$ compared with control mice of the same age (Figure 2, $\mathrm{B}$ and $\mathrm{C}$ ). $\mathrm{KO}$ mice had intermittent episodes of apnea (cycle time $\left[T_{\mathrm{TOT}}\right]$ exceeding twice the length of the mean) and periods during which $f R$ was very irregular, as measured by the irregularity score (IS) (24). These abnormalities increased with age $\left(r^{2}=0.50 ; P=0.02\right.$ for the number of apneas and for IS, $r^{2}=0.64 ; P<0.01$; Figure 2, D and E). In response to hypoxia ( 5 minutes at $10 \% \mathrm{O}_{2}$ ), there was an initial augmentation of breathing followed by a depression. Both neonate and older KO mice had equivalent augmentation of breathing, but much more severe depression in $V_{T}$ and $V_{E}$ compared with control mice (Figure $2 \mathrm{~F}$ ). Although variable, some $\mathrm{KO}$ mice also had an abnormal response to excess $\mathrm{CO}_{2}$ (hypercapnia; 5 minutes at $5 \% \mathrm{CO}_{2}$ ). Six out of 13 (neonate and adult) KO mice failed to show the hyperventilation response that was observed in all 6 control mice. Interestingly, the remaining $7 \mathrm{KO}$ mice had an exaggerated response compared with control mice (Supplemental Table 1).

We also measured breathing rate, heart rate, and arterial oxygen saturation in control and $\mathrm{KO}$ mice at various stages of disease progression by optical spectrometry in restrained animals. As with the plethysmography, breathing rate was variable, but with this method it was significantly lower in $\mathrm{KO}$ mice than in control mice $(\sim 150$ vs. $\sim 220$ breaths/min, KO vs. control; $P<0.001)$. This discrepancy between methods may be explained by the stress induced by restraint. Furthermore, the reduction in breathing rate was more evident in later stages of the disease (Figure 3, A and B, $P<0.05$ ). Likewise, heart rate for $\mathrm{KO}$ mice was lower than for control mice ( $\sim 500$ vs. $\sim 650$ beats $/ \mathrm{min}, \mathrm{KO}$ vs. control; $P<0.01)$, especially in late stages of the disease (Figure $3, \mathrm{C}$ and $\mathrm{D}, P<0.05$ ). Consistent with the lower breathing and heart rates, the percentage of satu- ration of arterial blood in late-stage $\mathrm{KO}$ mice was often less than $99 \%$, which was not observed in control mice ( $95 \%$ vs. 99\%, KO vs. control, $P<0.05$; Figure $3, \mathrm{E}$ and $\mathrm{F}$ ).

Abnormal responses of the pre-Bötzinger complex in $\mathrm{KO}$ mice. The preBötzinger complex (preBötC) in the ventral medulla is critical for respiratory rhythm generation $(25,26)$ and may play a role in the hypoxic response $(27,28)$. Extracellular recordings of brain stem slices containing the preBötC were obtained from P8-P10 KO mice and their control littermates. Under control conditions $\left(95 \% \mathrm{O}_{2}\right.$, $5 \% \mathrm{CO}_{2}$ ), these slices revealed no significant differences in frequency and regularity of bursting activity. In response to hypoxia $(95 \%$ $\mathrm{N}_{2}, 5 \% \mathrm{CO}_{2}$ ), the isolated preBötC generates an initial augmentation phase characterized by increased bursting frequency followed by a depression during which bursting frequency returns to, or falls below, baseline activity. During this response, the inspiratory burst discharge switches from "fictive eupnea" to "fictive gasping" (29). The initial augmentation phase in slices from KO mice did not differ from that of control littermates (Figure 4, A-F), but during depression, the amplitude of fictive gasping was significantly decreased in $\mathrm{KO}$ mice compared with controls (Figure 4, A-F). The frequency of fictive gasping was not statistically different between control and KO mice, but while $100 \%$ of slices from control mice continued fictive gasping throughout the 10 minutes of hypoxia, $30 \%$ of slices from $\mathrm{KO}$ mice stopped fictive gasping.

Extracellularly recorded population activity does not provide insight into how the amplitude was determined. Hence, we characterized the hypoxic response using intracellular recordings of preBötC inspiratory neurons in slices from $\mathrm{KO}$ and control mice. The cellular firing in phase with the extracellular burst was measured, and the number and frequency of action potentials per burst (APs/burst) was quantified. Under control conditions, the resting potential was identical between control and $\mathrm{KO}$ mice; however, the depolarization in phase with the network burst was significantly reduced in $\mathrm{KO}$ mice $(5.51 \pm 0.59 \mathrm{mV}, n=7)$ compared with controls $(10.13 \pm 1.30 \mathrm{mV}, n=5, \mathrm{p}<0.01$, Figure 4, G-I). AP/burst and firing frequency of $\mathrm{KO}$ mice were significantly lower than in control littermates (Figure 4, B and D). Upon transition from control to hypoxic conditions, the changes in resting potential in control and $\mathrm{KO}$ mice were similar. However, $\mathrm{KO}$ mice had a severe reduction of the underlying depolarization when challenged by hypoxia (from $5.51 \pm 0.59$ to $0.52 \pm 1.36 \mathrm{mV} ; n=7, P<0.01$ ), while control mice did not show a significant reduction $(10.13 \pm 1.30$ to $6.09 \pm 6.43 \mathrm{mV}$, Figure 4, G-I). The AP/burst of KO mice dropped dramatically, and 2 out of 7 of the slices stopped gasping after more than 5 minutes of hypoxia, while in control slices, a relatively mild reduction of AP/burst was observed, and all of the control slices maintained gasping (Figure 4, C and E). Interestingly, the lack of gasping observed in $\mathrm{KO}$ slices in hypoxic conditions was restored after bath application of tolbutamide, an ATP-sensitive potassium channel $\left(\mathrm{K}_{\mathrm{ATP}}\right)$ inhibitor (Supplemental Figure 1), suggesting an intrinsic deficit in the physiology of preBötC neurons, which may contribute to the breathing alterations in $\mathrm{KO}$ mice.

Although respiratory impairment is one of the clinical signs of $\mathrm{KO}$ mice, microglial activation was rarely observed in any of the classical respiratory centers, such as the medial parabrachial nucleus, nucleus of the solitary tract, or preBötC. However, in late-stage $\mathrm{KO}$ mice, microglial activation was observed around serotonergic cell bodies in the medullary raphe (raphe pallidus and obscurus), a brain region important for respiratory control (30). In some cases, microglial activation extended to their projections, accompanied by 
A

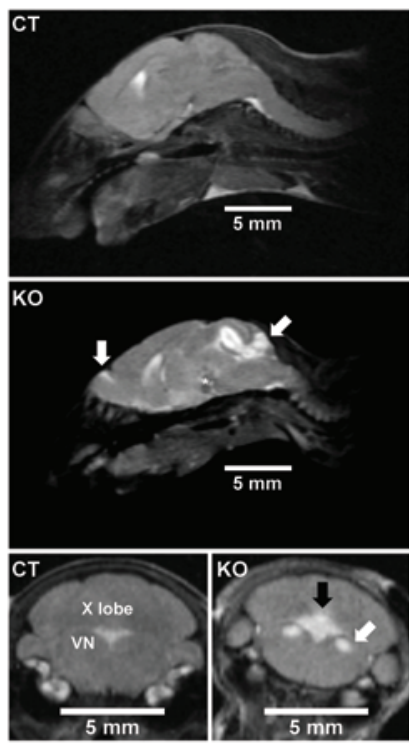

B

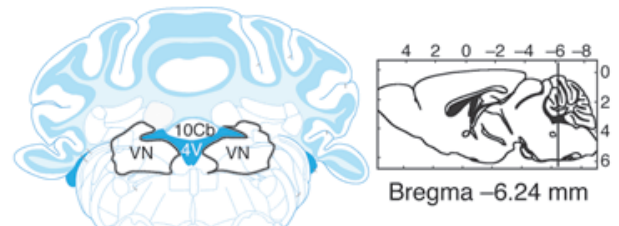

C

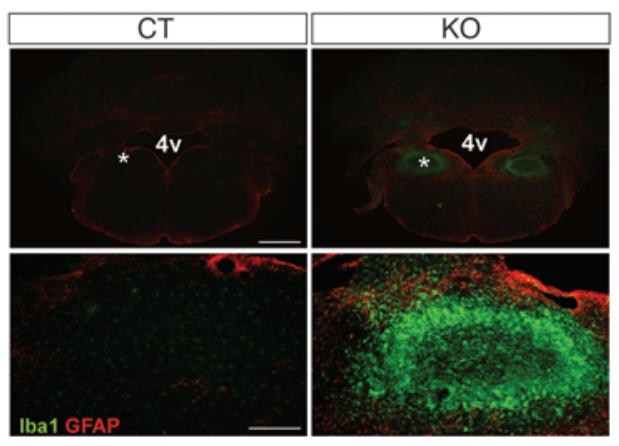

D

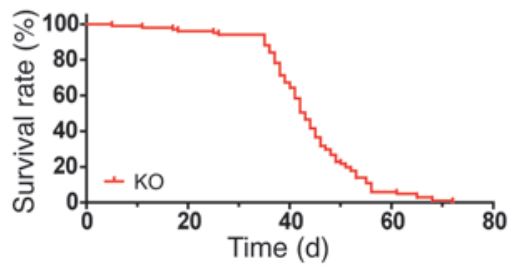

\section{Figure 1}

Alterations in MRI and histopathology in KO mice. (A) T2-weighted in vivo MRI of control and late-stage KO mouse. Sagittal MRIs (top panels) of control (CT) mouse and late-stage KO mouse show hyperintense regions (white areas) in lateral ventricles of control mouse, whereas KO mouse shows distinct lesions in exterior plexus of $\mathrm{OB}$ and dorsal surface of brain stem with extensive lesions of cerebellum (white arrows) together with a hypointense alteration in the midbrain (asterisk). Coronal imaging of control and $\mathrm{KO}$ mice shows only moderate T2-weighted MRI intensity in fourth ventricular region of control mouse whereas a mid-stage $\mathrm{KO}$ mouse (bottom, right) had significant lesions of the dorsal medulla (VN, white arrow) and tenth cerebellar lobe (black arrow). (B) Diagram of the VN according to Franklin and Paxinos (60). VN is circled. 4v, fourth ventricle. (C) Staining for lba1 (microglial cell marker) and GFAP (astrocyte marker) in the $\mathrm{VN}$ of control (top left) and KO (top right) mice shows presence of symmetrical lesions filled with abundant microglial cells and surrounded by GFAP-positive reactive astrocytes in KO compared with control mice. Scale bar: 1,000 $\mu \mathrm{m}$. (Bottom row) Close-up images of the VN (indicated by asterisks in C) show increased GFAP and lba1 intensity and morphological changes in glial cells in KO mice. Scale bar: $250 \mu \mathrm{m}$. (D) Survival curve of KO mice $(n=101)$.

mild microgliosis in the preBötC that was identified by neurokinin 1 receptor immunolabeling (Supplemental Figure 2, A-D), suggesting that the alterations in these neurons was not sufficient to elicit a severe secondary inflammatory response. No degenerative changes were detected in the chemoreceptive Phox2B-immunoreactive neurons of the retrotrapezoid nucleus $(31,32)$.

Selective inactivation of Ndusf4 in the VN induces neurodegeneration and breathing abnormalities and increases mortality. The preBötC is essential for breathing in vivo (25), and it is influenced by a large number of neural inputs including those from the VN (see Discussion). Because the $\mathrm{VN}$ is a prominent site of gliosis and neurodegeneration in $\mathrm{KO}$ mice, we selectively inactivated the $N d u f s 4$ gene only in that nucleus by bilaterally injecting adult $N d u f 4^{l o x} /$ lox mice with adenoassociated virus (type 1) expressing CreGFP (AAV1-Cre-GFP) to generate AAV-VN-KO mice. As a control, $N d u f 4^{l o x} / / 0 x$ mice were injected with an AAV1 encoding a nonfunc- tional Cre (AAV1-Cre $\Delta$-GFP) to generate AAV-VN-CT mice. Extensive GFP expression was observed within the $\mathrm{VN}$ after bilateral viral transduction (Figure 5A). Furthermore, AAV-VN-KO mice developed extensive microglial activation 1 month after surgery, assessed by staining of the Iba1 marker (Figure 5A). Iba1 staining was dependent upon loss of $N d u f s 4$ expression because no remarkable microglial activation was observed in the $\mathrm{VN}$ of AAV-VN-CT mice (Figure 5A).

Inactivation of $N d u f s 4$ bilaterally within the VN led to weight loss (Figure 5C), motor coordination deficits as assessed by rotarod testing (Figure 5D), and increased mortality (Figure 5B). As the behavior of the mice deteriorated, we observed occasional gasping by the AAV-VN-KO mice. Plethysmography revealed that they had a normal response to hypoxia (5 minutes with $10 \% \mathrm{O}_{2}$ ) (Figure 5E), but a blunted hypercapnic ( 5 minutes with $5 \% \mathrm{CO}_{2}$ ) ventilatory response (Figure 5F). Moreover, AAV-VN-KO mice had more irregular breathing (IS $=21.2 \pm 2.30 \mathrm{AAV}-\mathrm{VN}-\mathrm{CT}$ mice, $n=14 \mathrm{vs}$. IS $=36.6 \pm 6.10 \mathrm{AAV}-\mathrm{VN}-\mathrm{KO}$ mice, $n=7 ; P<0.05)$. Injection of the AAV1-Cre-GFP into the VN of conditional tdTomato reporter (line Ai14) mice (33) did not produce any of the symptoms observed when the virus was injected into $N d u f s 4^{l o x} / l o x$ mice (not shown). Analysis of the Cre-induced tdTomato fluorescence from the reporter gene confirmed that transductions of cells was restricted to the $\mathrm{VN}$ and that this nucleus sends projections to the ventral medulla in the vicinity of the preBötC (Supplemental Figure 3) as described before (34).

Viral restoration of $\mathrm{Ndufs} 4$ in the $V N$ attenuates respiratory abnormalities and ameliorates the fatal phenotype of $\mathrm{KO}$ mice. Because loss of $N d u f s 4$ function in the VN contributes to the motor phenotype and breathing abnormalities of $\mathrm{KO}$ mice, we asked whether reexpression of $N d u f s 4$ in the VN was sufficient to ameliorate disease progression in $\mathrm{KO}$ mice. An AAV1 construct encoding Ndufs4-IRES-GFP was delivered bilaterally into the $\mathrm{VN}$ of $\mathrm{KO}$ mice at P21 as a viral rescue strategy (referred to as AAV-VN-VR mice). At P21, the mice were small ( $<15 \mathrm{~g})$, presymptomatic $(22,23)$, and more sensitive to anesthesia, making viral injections challenging. Correct targeting was confirmed by extensive GFP expression within the VN (Figure 6A). In accordance with our previous work (23), histopathological analysis of the $\mathrm{VN}$ of noninjected mice showed severe microglial activation and tissue lesions (Figure 6B) that were markedly reduced in the injected mice (Figure 6C), suggesting that the cell death observed in the $\mathrm{VN}$ of noninjected $\mathrm{KO}$ mice is due to the lack of Ndufs 4 in that nucleus. The viral rescue delayed clinical progression of the disease (Figure 6F, $P<0.05$ ) and significantly increased the life span of AAV-VN-VR mice compared 
A $\mathrm{CT}$
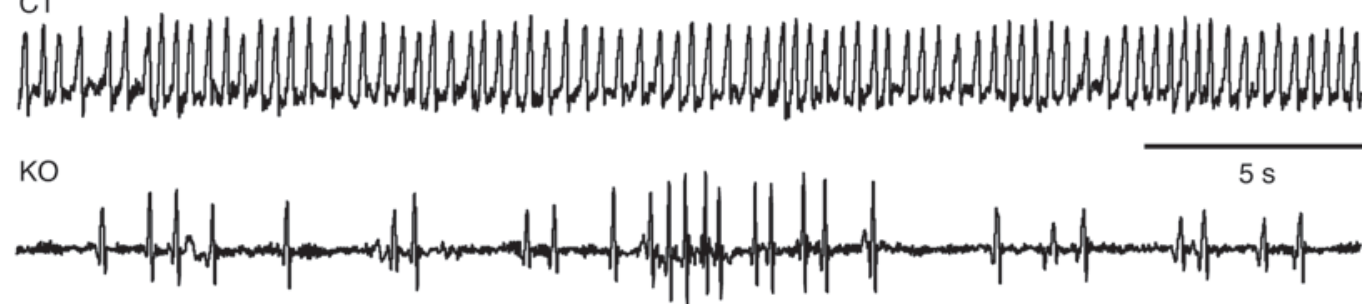

B Neonates
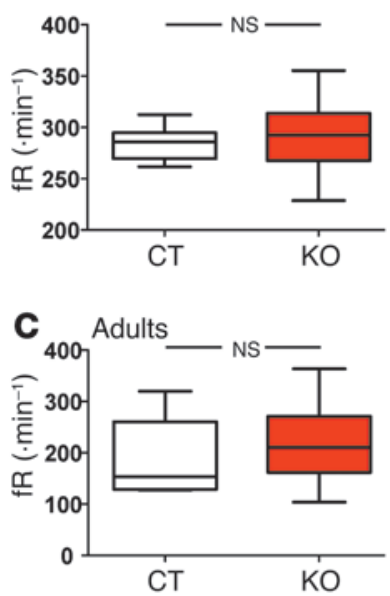

F

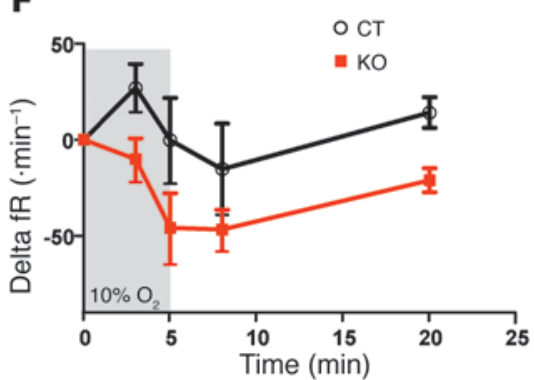

D

CT
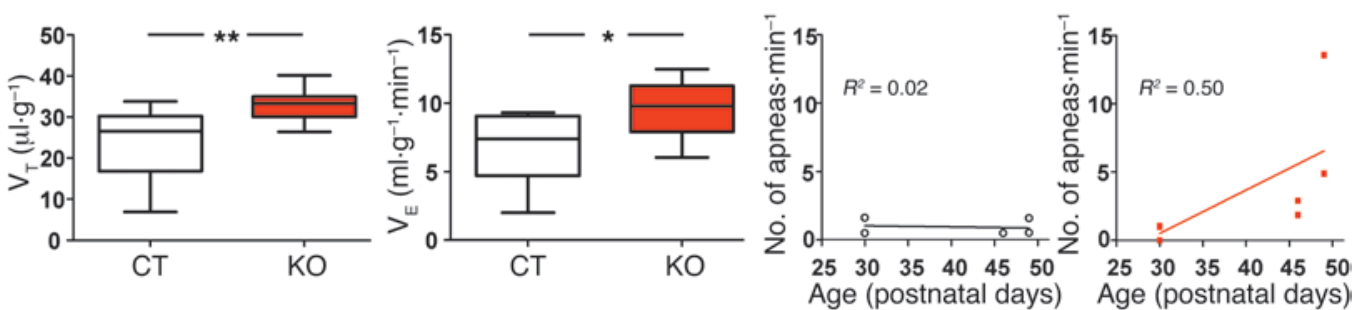

E
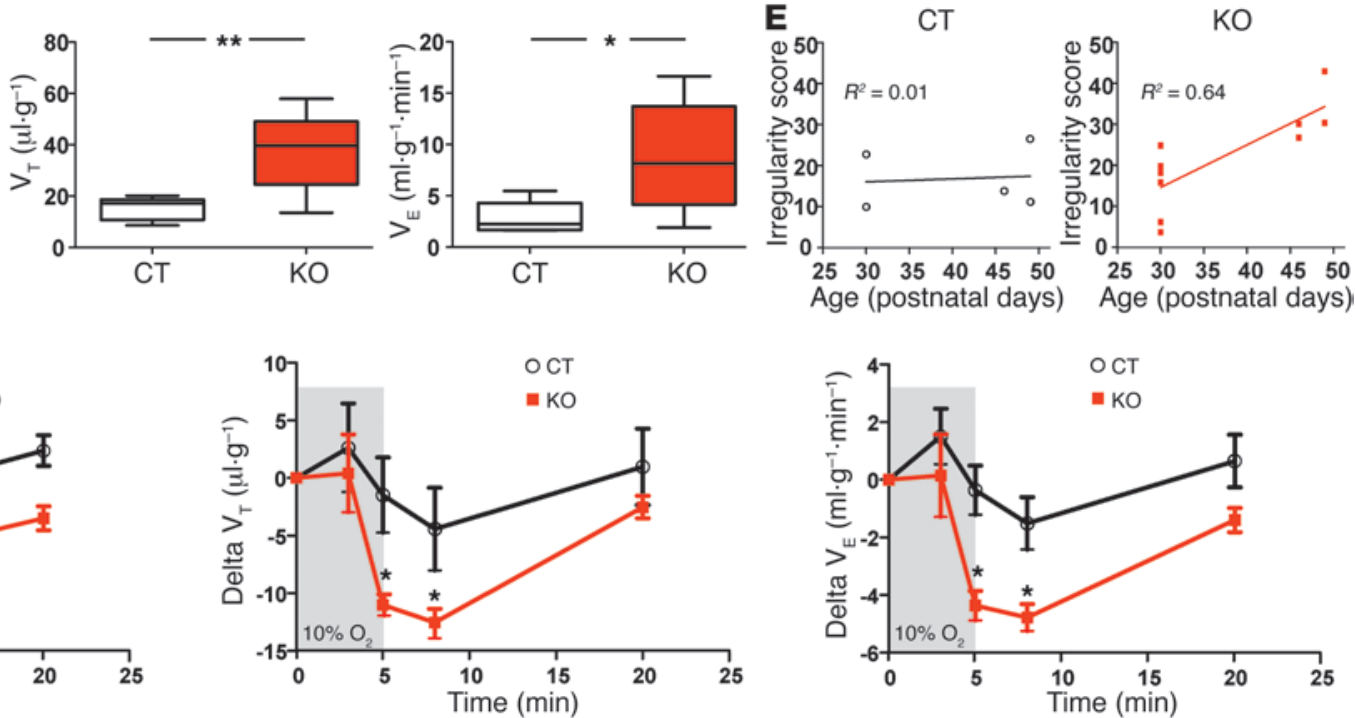

Figure 2

Abnormal breathing patterns of KO mice. (A) A 30-second breathing record of freely moving, 46-day-old mice recorded by barometric, whole-body, flow plethysmography reveals breathing irregularity and apneas in the $\mathrm{KO}$ mouse (bottom trace) compared with the control littermate (top trace). Upward deflections in traces represent inspiratory movements. (B and $\mathbf{C})$ Breathing parameters of neonate (B) and adult (C) control and KO mice. The $V_{T}$ and $V_{E}$ are significantly higher in KO mice than in control mice. ${ }^{*} P<0.05$; ${ }^{* *} P<0.01$, Student's $t$ test. (D and E) Development of apneas (D) and irregularity of the breathing frequency $(\mathbf{E})$ of control and $\mathrm{KO}$ mice were significantly correlated with age. (F) Changes in breathing of neonate control and $\mathrm{KO}$ mice exposed to 5 minutes of hypoxia $\left(10 \% \mathrm{O}_{2}\right.$; gray area). The respiratory depression of $\mathrm{KO}$ mice observed at the fifth minute of exposure was significantly greater than that of control mice, ${ }^{*} P<0.01,2$-way ANOVA, Bonferroni's post-hoc test. Data are shown as mean \pm SEM.

with $\mathrm{KO}$ mice (Figure 6G; median life span: 53 days for KO vs. 69 days for AAV-VN-VR; $P<0.01)$. Interestingly, the longer-lived AAV-VN-VR mice, which had reduced gliosis in the VN, developed lesions in the striatum (Figure 6, D and E). Striatal lesions are common in people with LS and other mitochondrial diseases, but had not been seen before in late-stage (P50) KO mice (23), suggesting that the prolonged life span observed in the virally rescued mice reveals other brain regions where gliosis develops more slowly.

To ascertain whether improved respiratory response may account for the reduced mortality in the AAV-VN-VR mice, we measured the response of late-stage virally rescued $\mathrm{KO}$ mice and controls (control mice injected with AAV-Cre $\Delta$-GFP) by plethysmog- raphy. AAV-VN-VR mice showed a breathing response to hypoxia comparable to that of control mice during the early (third minute after of hypoxia) phase of the response. However, this was lost at later time points, with the AAV-VN-VR mice showing a response parallel to that of $\mathrm{KO}$ mice (Figure $6 \mathrm{H}$ and Supplemental Figure 4 , A and B). Restoring expression of Ndufs 4 in the VN also normalized the hypercapnic ventilatory response of the AAV-VN-VR mice compared with virally injected control mice, in contrast with that of hyperresponding or nonresponding KO mice (Figure 6I and Supplemental Figure 4, C and D). Furthermore, the breathing of the AAV-VN-VR mice recovered its regularity (IS $=21.2 \pm 2.30$ for control mice, $n=14$; IS $=25.7 \pm 4.43$, for AAV-VN-VR, $n=3$ ). 

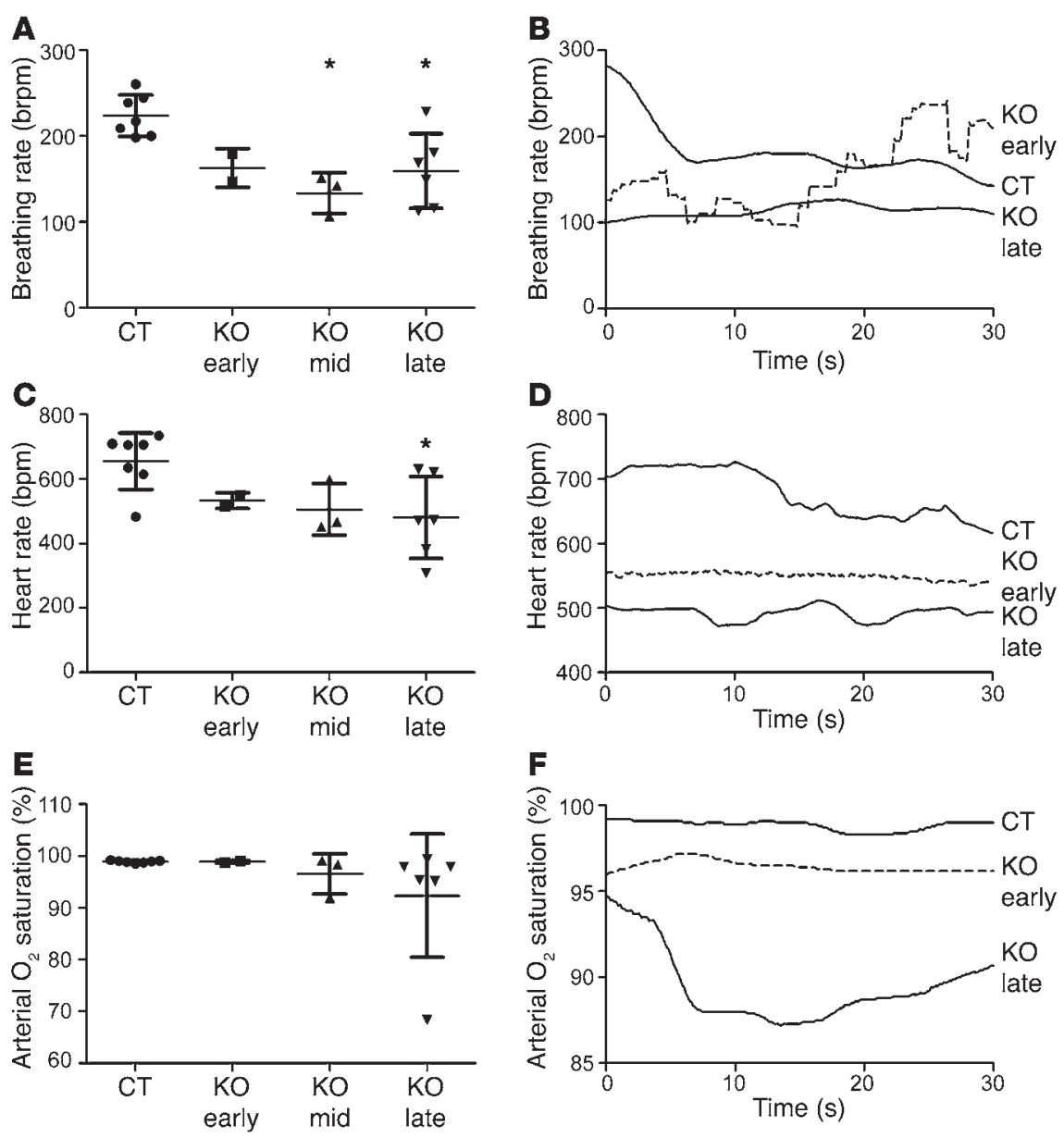

\section{Figure 3}

Reduced breathing, heart rate, and oxygen saturation in $\mathrm{KO}$ mice. Measurements were made using a pulse oximeter attached to the bare necks of control $(n=7)$ and KO mice at various stages of disease progression (total $n=11 ; n=2$, 3, and 6 mice, early, mid-stage, and late-stage respectively) as described in Methods. For KO mice, there were often significant fluctuations of the values over time; average rates during the measurement period are plotted. (A) Breathing rate was lower in mid- and late-stage KO mice. ${ }^{*} P<0.05$, 1-way ANOVA, Tukey's post-test. (B) Representative traces of control and early- and late-stage KO mice $(\mathbf{C})$ Heart rate at all stages of disease progression was significantly less than that of controls. ${ }^{*} P<0.05$, 1-way ANOVA, Tukey's post-test. (D) Representative traces of control and early- and late-stage KO mice. (E) Arterial blood oxygen saturation became variable and significantly lower in late-stage $\mathrm{KO}$ mice. (F) Representative traces of control and early- and late-stage KO mice. Data points represent individual mice. Data are shown as mean \pm SEM.
These results show that restoring Ndufs 4 function to the $\mathrm{VN}$ is sufficient to partially correct the early breathing deficits in $\mathrm{KO}$ mice and ameliorate the fatal disease progression of $\mathrm{KO}$ mice.

\section{Discussion}

A primary focus of the present study was to elucidate the probable cause of death of $\mathrm{KO}$ mice. We started with knowledge of the brain regions that showed the most gliosis and neurodegeneration, as revealed by histopathology and MRI, but the observation of gasping behavior of $\mathrm{KO}$ mice provided the first indication of abnormal breathing behaviors. We noted previously (23) that $\mathrm{KO}$ mice resemble humans with LS with regard to a large range of phenotypes and we now extend that to breathing. Respiratory abnormalities affect many LS patients $(8,9,35,36)$ and virtually all patients with mutations in the NDUFS4 gene (16-21,37); however, the link between mitochondrial dysfunction and breathing could not be investigated without an animal model.

We propose that lesions within the $\mathrm{VN}$ and $\mathrm{FN}$ as well as compromised preBötC function in $\mathrm{KO}$ mice result in dysregulation of the central respiratory network and ultimately respiratory failure. Breathing rates determined by optical spectrometry were variable, but older KO mice displayed a reduced breathing rate, especially under stress, along with lower heart rates and incomplete oxygenation of arterial blood compared with control mice. Both neonate and adult $\mathrm{KO}$ mice had higher ventilation and higher $V_{T}$ compared with control mice, as measured by plethysmography, which may be a compensatory response. Patients with LS also display hyperventilation and hypocapnia, resulting in respiratory alkalosis (38). Breathing by KO mice became more irregular and was interrupted by frequent apneas as they grew older. Apneas were a common response to handling. $\mathrm{KO}$ mice had abnormal responses when exposed to low levels of $\mathrm{O}_{2}$ or high levels of $\mathrm{CO}_{2}$. Although changes in oxygen and $\mathrm{pH}$ are thought to be primarily detected in the arterial blood by the carotid bodies (39), the CNS is also sensitive to changes in central levels of $\mathrm{O}_{2}$ and $\mathrm{CO}_{2}(27,31$, $40,41)$. In the absence of carotid bodies, hypoxia still triggers a ventilatory response (42). Furthermore, mice lacking $N d u f s 4$ function only in the CNS have identical breathing defects; therefore, central rather than peripheral mechanisms are primarily involved. Indeed, brain stem slices containing the preBötC exhibited severe abnormalities in inspiratory bursting activity. Under normoxic conditions, inspiratory neurons of KO mice generated fewer APs per burst, which was associated with a lower firing frequency rate compared with that of control mice. These results suggest that the increased $V_{T}$ and $V_{E}$ in the intact animal is a compensatory mechanism to a decreased central respiratory drive. When challenged with a 10-minute exposure to hypoxia, the amplitude of fictive gasping in $\mathrm{KO}$ mice was reduced compared with that in controls, although the initial augmentation phase was identical. Hence, the central respiratory network initially compensates for decreased levels of $\mathrm{O}_{2}$, but fails when decreased levels are maintained for a long time, which may explain why $\mathrm{KO}$ mice generate 

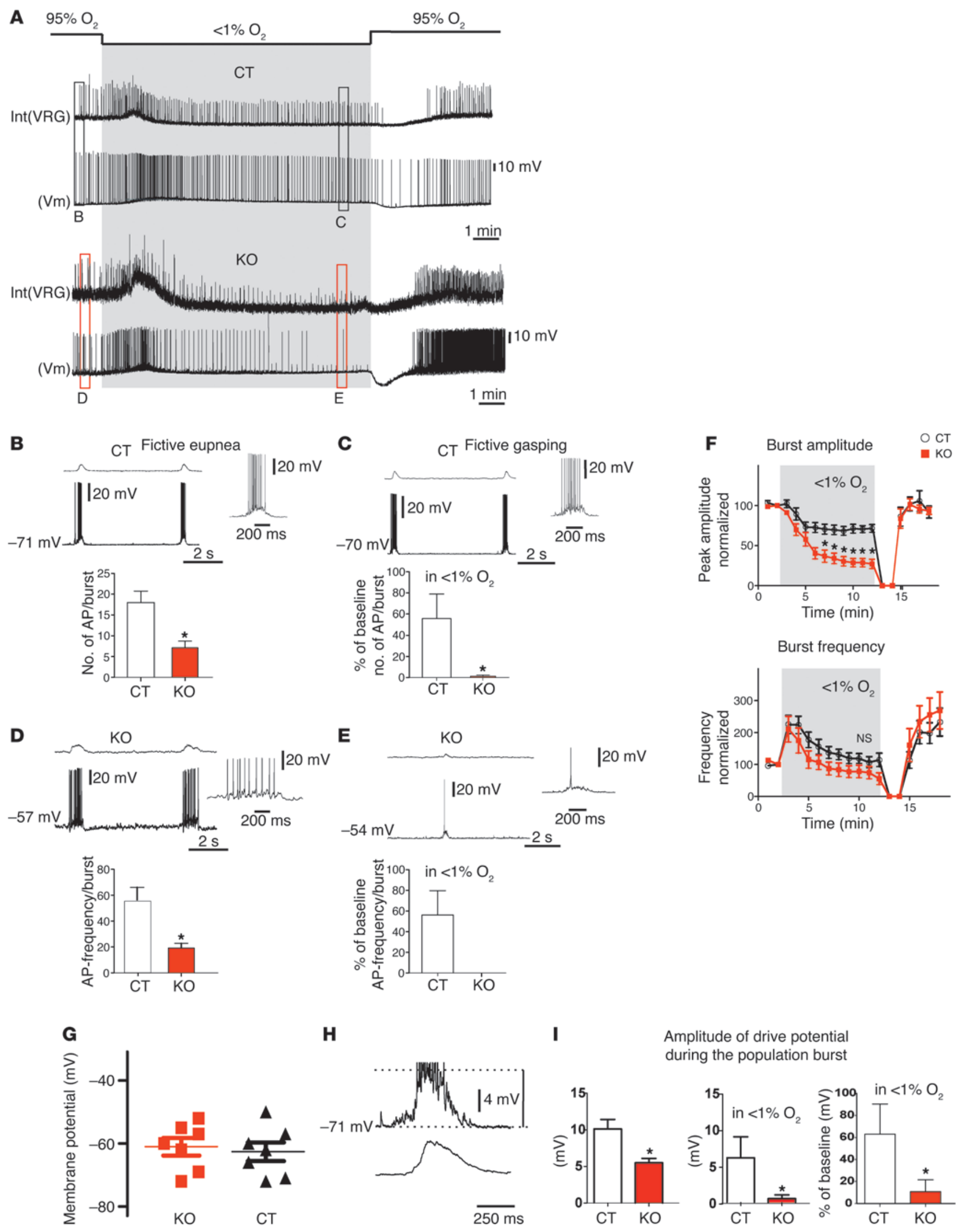

Amplitude of drive potential during the population burst 


\section{Figure 4}

Abnormal extra- and intracellular recordings of PreBötC of KO mice. (A-E) Simultaneous intracellular whole cell (lower trace, whole-cell voltage; $\mathrm{Vm}$ ) and multi-unit population recordings (upper trace, integrated and rectified multi-unit recording; VRG) containing PreBötC from brain stem slices of $\mathrm{KO}$ and control mice in response to hypoxic conditions $\left(95 \% \mathrm{~N}_{2} / 5 \% \mathrm{CO}_{2}\right.$, A). Intracellular recordings during fictive eupnea revealed a lower number of APs/burst (B) and a lower AP frequency (D) of KO mice $(n=8)$ compared with control mice $(n=5)$. In hypoxic conditions, control mice decreased to $56.96 \% \pm 47.04 \%$ of baseline $(\mathbf{C}, n=5)$, while KO mice $(\mathbf{E}, n=8)$ stopped firing APs by the end of the 10-minute hypoxic exposure. (F) Population recordings of KO mice $(n=10)$ showed significantly $(P<0.05)$ reduced fictive gasping compared with control mice $(n=10)$. $(\mathbf{G})$ Resting potential of inspiratory cells was not significantly different in slices from KO mice compared with control mice. Data points represent individual cells. (H) Example of a drive potential (upper trace, whole cell voltage; $\mathrm{Vm}$ ) during a population burst (lower trace, integrated and rectified multi-unit recording of the VRG). (I) The depolarization in phase with the network burst was significantly reduced in slices from $\mathrm{KO}$ mice (5.51 $\pm 0.59 \mathrm{mV}$, $n=7)$ when compared with the control mice $(10.13 \pm 1.30 \mathrm{mV}, n=5, P=0.005)$ under normoxic conditions. In addition, the KO mice had a severe reduction of the underlying depolarization when challenged by hypoxia (from $5.51 \pm 0.59$ to $0.52 \pm 1.36 \mathrm{mV}, n=7, P=0.0012$ ), while the control mice did not show a significant reduction $(10.13 \pm 1.30 \mathrm{mV}$ to $6.09 \pm 6.43 \mathrm{mV}) .{ }^{*} P<0.01$. Data are shown as mean \pm SEM.

more apneas with increasing age. Increased apneas and blunted ventilatory responses to hypoxia and hypercapnia may cause fatal respiratory arrest, as suggested for other pathologies $(43,44)$. In agreement with our results, all described NDUFS4-LS patients presented apneic episodes and died of cardiorespiratory failure (21). KO mice, however, also presented altered heart rate, especially at older ages, which could contribute to the death of the animals. However, the neural origin of $\mathrm{KO}$ pathology has been established previously, and deletion of $N d u f s 4$ in cardiomyocytes and skeletal muscle do not cause any overt clinical phenotype in mice (45), ruling out a primary cardiac defect. Although defi- cits in the peripheral nervous system inputs to the heart or heart conductivity were not tested in our model, they have not been consistently reported in LS patients $(7,21)$.

ATP is involved in the maintenance of gasping during the depression phase (46). Furthermore, during hypoxia, ATP-sensitive potassium channels have been reported to be activated in inspiratory neurons of the preBötC (47); therefore, reduced ATP generation may play a major role in this response. Interestingly, mice lacking ATPsensitive potassium channel 6.2 showed an impaired maintenance of gasping during severe hypoxia (48). We hypothesize that $\mathrm{KO}$ mice produce less ATP in energy-demanding cells because of complex I
A
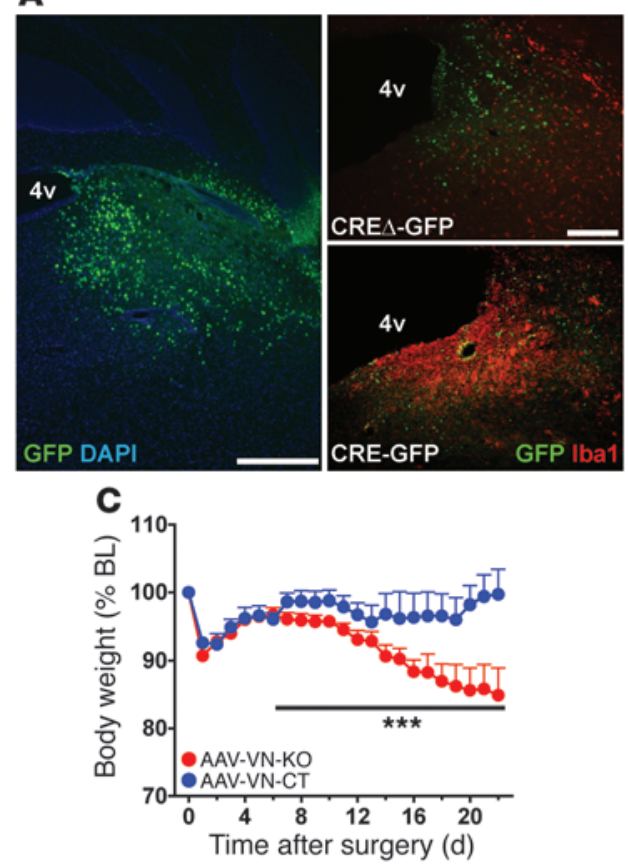

E

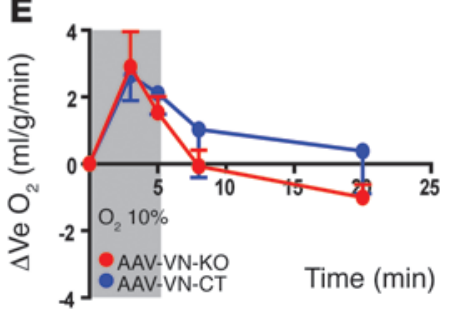

B

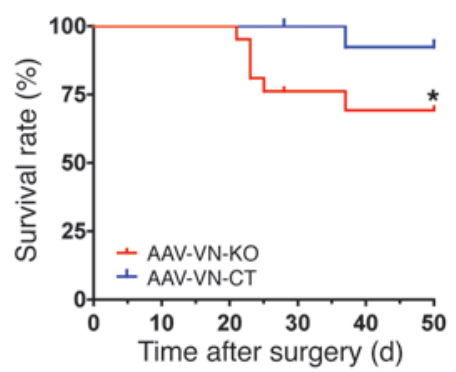

\section{Figure 5}

Histopathological and behavioral alterations after Ndufs4 inactivation in the VN. (A) (Left panel) Representative image of the degree of transduction (GFP-positive cells) after vector delivery in the VN. (Right panels) Iba1 staining (red) after injection of a nonfunctional Creexpressing (CRE $\Delta$-GFP, AAV-VN-CT mice, top right) or a functional Cre-expressing (CRE-GFP, $A A V-V N-K O$ mice, bottom right) AAV1 vector in the VN of Ndufs 4lox/lox mice. Scale bars: $500 \mu \mathrm{m}$ (left panel); $250 \mu \mathrm{m}$ (right panels). (B) Survival curve in AAV-VN-CT $(n=17)$ and AAV-VN-KO mice $(n=21)$ after $V N$ injection. ${ }^{*} P<0.05$, GehanBreslow-Wilcoxon Test versus AAV-VN-CT. (C) Percentage of body weight change after injection in AAV-VN-CT $(n=10)$ and AAV-VN-KO $(n=15)$ mice. Day 0 is considered baseline (BL) (100\%). ${ }^{* *} P<0.001$, genotype, 2-way ANOVA. (D) Change in rotarod performance after injection in AAV-VN-CT $(n=6)$ and AAV-VN-KO $(n=7)$ mice. ${ }^{*} P<0.05$; ${ }^{* *} P<0.01,2$-way ANOVA, Bonferroni's post-hoc test versus AAV-VN-CT. (E) Changes in breathing of AAV-VN-CT $(n=14)$ and AAV-VN-KO $(n=6)$ mice exposed to 5 -minute hypoxia $\left(10 \% \mathrm{O}_{2}\right.$; gray area). ( $\left.\mathbf{F}\right)$ Changes in breathing of AAV-VN-CT $(n=14)$ and AAV-VN-KO $(n=6)$ mice exposed to 5-minute hypercapnia (5\% $\mathrm{CO}_{2}$; gray area). ${ }^{*} P<0.05 ;{ }^{* *} P<0.01$ versus AAV-VN-CT; 2-way ANOVA, Bonferroni's posthoc test. Data are shown as mean \pm SEM. 

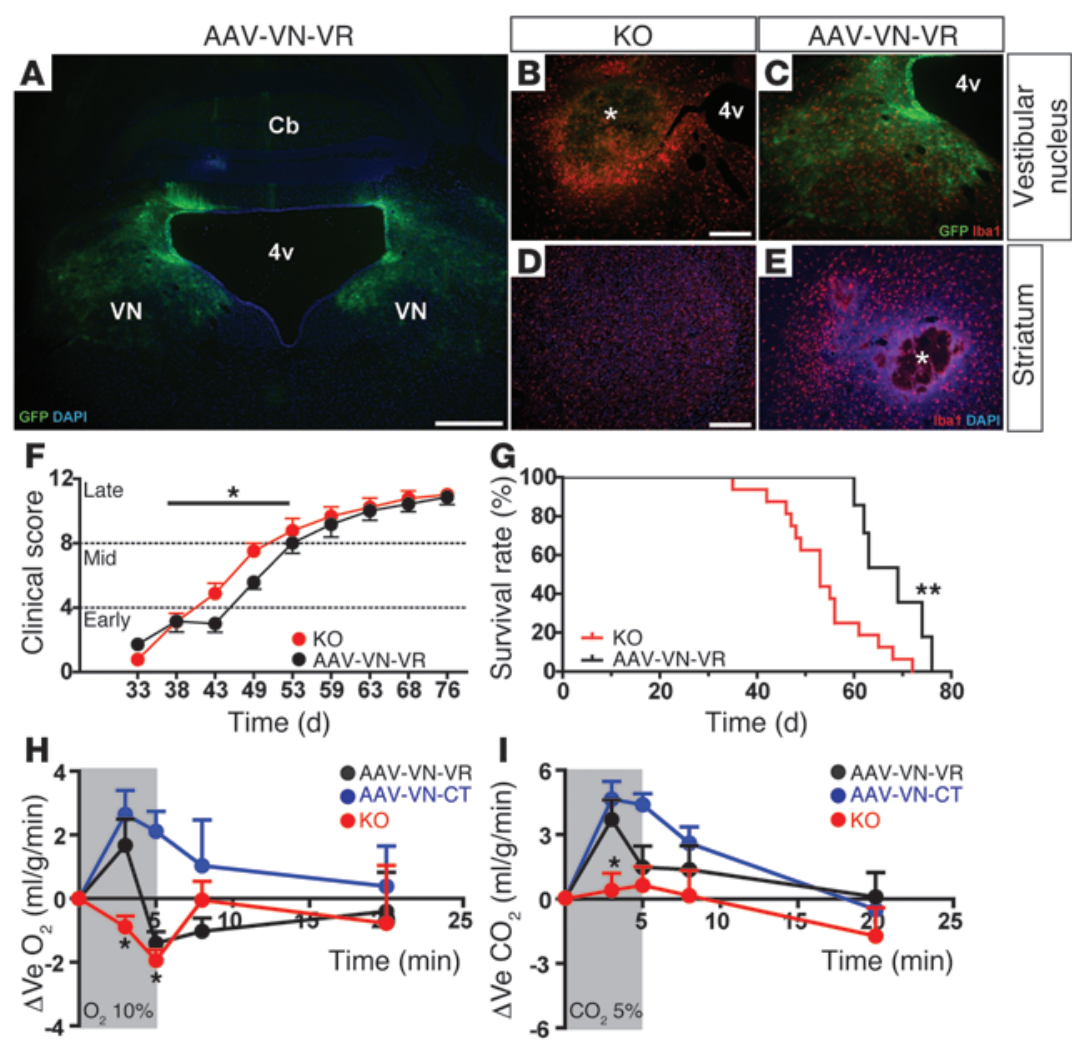

\section{Figure 6}

Ndufs 4 reexpression in the $\mathrm{VN}$ of $\mathrm{KO}$ mice improves breathing and ameliorates disease progression. (A) Representative image of the degree of transduction (GFP-positive cells) after vector delivery in the VN of $K O$ mice (AAV-VN-VR mice). (B-E) Iba1 staining (red) in the VN (B and $\mathbf{C}$ ) or striatum (D and $\mathbf{E}$ ) of $\mathrm{KO}$ or AAV-VN-VR mice. Asterisk denotes presence of lesions. $\mathrm{Cb}$, cerebellum. Scale bars: $500 \mu \mathrm{m}$ (A); $250 \mu \mathrm{m}$ (B-E). (F) Clinical score of the disease progression in $\mathrm{KO}(n=9)$ and AAV-VN-VR $(n=7)$ mice as described (24). ${ }^{*} P<0.05$ genotype, 2-way ANOVA. $F(1,121)=4.18$. (G) Survival curve in KO $(n=16)$ and AAV-VN-VR $(n=7)$ mice. ${ }^{* *} P<0.01$, Gehan-Breslow-Wilcoxon test versus $\mathrm{KO}$ mice. (H) Changes in breathing of AAV-VN-CT $(n=14)$, AAV-VN-VR $(n=3)$, and KO $(n=10)$ mice exposed to 5 -minute hypoxia $\left(10 \% \mathrm{O}_{2}\right.$; gray area). ${ }^{*} P<0.05$ versus control mice, 2 -way ANOVA, Bonferroni's post-hoc test. (I) Changes in breathing of AAV-VN-CT $(n=14)$, AAV-VN-VR $(n=3)$, and nonresponding $\mathrm{KO}(n=5)$ mice exposed to 5-minute hypercapnia $\left(5 \% \mathrm{CO}_{2}\right.$; gray area). ${ }^{*} P<0.05$ versus control mice, 2-way ANOVA, Bonferroni's post-hoc test. Data are shown as the mean \pm SEM. dysfunction. Therefore, during hypoxia, when the ability to produce ATP is compromised, the ATP levels of cells in KO mice may drop dramatically compared with those in control mice, leading to an impaired gasping response which may lead to opening of $\mathrm{K}_{\mathrm{ATP}}$ channels. Accordingly, bath application of a $\mathrm{K}_{\mathrm{ATP}}$ channel antagonist restored fictive gasping during hypoxic events in PreBötC slices of $\mathrm{KO}$ mice. Further work is warranted to confirm this hypothesis.

Although our results indicate that intrinsic properties of preBötC are compromised in $\mathrm{KO}$ mice, the mild gliosis within this complex suggests that regulatory inputs to the preBötC play an important role in respiratory dysfunction and death. The cerebellum, $\mathrm{FN}$, and $\mathrm{VN}$ are responsible for maintaining balance as well as modulation of breathing, coordination of breathing and swallowing, and adjustment of breathing to postural changes (49). The importance of the cerebellum and brain stem for regulation of automatic ventilation is also manifest in sleep apneas (50-52). These structures also mediate autonomic breathing dysfunction in sudden infant death syndrome and other sleep disorder breathing and cardiovascular syndromes $(51,53)$. Electrical stimulation of $\mathrm{VN}$ neurons modulates firing properties of neurons in the preBötC $(34,54)$. Consequently, we hypothesize that the gradual demise of the $\mathrm{VN}$ network contributes to both the ataxia and respiratory abnormalities observed in $\mathrm{KO}$ mice.

Inactivation of $N d u f s 4$ selectively in the $\mathrm{VN}$ leads to abnormal control of breathing, and viral restoration of $N d u f s 4$ expression in the $\mathrm{VN}$ ameliorates the breathing deficits and mortality of $\mathrm{KO}$ mice. Thus, Ndufs 4 function in the $\mathrm{VN}$ is critical for maintenance of normal balance and ventilation in response to hypercapnia. KO mice with $N d u f s 4$ restored only in the VN probably still have defects in other regulatory inputs to the preBötC as well as the preBötC itself. Furthermore, our viral injections were in the medial and lateral parts of the $\mathrm{VN}$ because they show the most gliosis (23); however, other $\mathrm{VN}$ subdivisions may also be involved in breathing (55). Other neighboring structures in the dorsal medulla or axons coursing through this area may be affected by inflammation and edema. For example, the nucleus tractus solitarius is involved in breathing regulation (56), and it (along with the $\mathrm{VN}$ ) projects to the parabrachial nucleus. Lesions of the parabrachial nucleus promote motion sickness, nausea, and anorexia, which are common conditions associated with breathing irregularities and are often observed in LS patients (7).

In summary, our studies validate the usefulness of $\mathrm{KO}$ mice as a model for studying the physiological consequences of mitochondrial dysfunction in the brain and underscore the importance of the $\mathrm{VN}$ in disease progression.

\section{Methods}

Mice. Mice were maintained with rodent diet (5053; PicoLab) and water available ad libitum in a vivarium with a 12 -hour light/12-hour dark cycle at $22^{\circ} \mathrm{C}$. The $N d u f s 4-\mathrm{KO}$ and $N d u f 4^{40 x} /$ lox mice were bred as described (22) and backcrossed onto a C57BL/6 background for more than 10 generations. The NesKO mice were generated by breeding $N d u f s^{4 l o x} /$ lox mice with B6. Cg(SJL)- $\mathrm{Tg}$ (Nes-cre) $1 \mathrm{Kln} / \mathrm{J}$ (Nestin-Cre) mice obtained from Jackson Laboratory. Because KO and NesKO mice present an identical encephalopathy progression (23), they are used interchangeably for the studies presented here and referred to as KO mice. The (Gt)ROSA26Sor-floxed stoptdTomato (Ai14) line (33) was obtained from the Allen Institute for Brain Science (Seattle, Washington, USA).

Clinical scoring. Disease progression was evaluated as described (23). Briefly, physiological (body weight, body temperature, breathing pattern) and behavioral (locomotor activity, motor coordination, gait/postural alterations) parameters were evaluated as follows: cumulative scores of $0-1$, presymptomatic stage; $2-4$, early stage; $5-8$, middle stage; $9-12$, late stage. 
$A A V$-based vector generation and delivery. AAV-CRE-GFP and AAV-CRE $\triangle$ GFP have been described elsewhere $(57,58)$. An AAV-Ndufs4-IRES-GFP vector was generated by cloning the mouse $N d u f s 4$ gene in an IRES-GFP containing plasmid under the control of the CMV-chicken $\beta$-actin (CBA) promoter. Viruses were prepared in HEK293 cells with AAV1 coat serotype, purified by sucrose and CsCI gradient centrifugation steps, and then resuspended in HBSS at a titer of $2 \times 10^{9}$ viral genomes $/ \mu \mathrm{l}$. For viral delivery, $1 \mu \mathrm{l}$ of the virus was stereotaxically injected in the mediolateral $\mathrm{VN}$ at the coordinates (mediolateral $[\mathrm{ML}]= \pm 1.25$, anterioposterior $[\mathrm{AP}]=-6.00$, dorsoventral $[D V]=-3.90)$ from the bregma. Viruses were injected bilaterally and animals recovered for 2 weeks before behavioral testing.

MRI. Mice were anesthetized with $1.5 \%$ isoflurane and maintained at $37^{\circ} \mathrm{C}$ during the experiment with forced warmed air and on a heating pad. During the MRI session, the mouse was mounted in a coil such that the signal was focused on the head region. The mouse was held in position with several pieces of adhesive tape. Approximate parameters used were as follows: spin echo-type parameters $3000 / 40 \mathrm{~ms}$ cycle delay/echo time, 2 -average, 12 -minute acquisition time. After imaging, the mice were monitored to assure full recovery before returning to the cage.

Pulsimetry and oximetry by optical spectroscopy. The hair in the neck region of mice was removed with Nair depilatory cream using a 5-minute application; cream was then thoroughly removed with moist paper towel. At least 24 hours later, oxygen saturation, breathing rate, and heart rate were measured using the MouseOx (Starr Life Sciences) optical spectroscopy apparatus and software. After 5 minutes of acclimatization to being restrained by hand, a collar clip was placed around the neck of the mouse. Recordings were made for 10 to 20 minutes. During recording, slight movements or renewal of struggling often caused loss of signal or error in one or more parameters. Only time periods containing a completely error-free signal for each measured parameter were used for analyses. For each mouse, at least 6 different time points lasting at least 6 seconds were collected for a total of at least 1 minute of data, which were later used for statistical analyses.

Whole-body plethysmography. Ventilatory function was assessed by wholebody plethysmography under unrestrained conditions (Buxco Research System). Briefly, normoxic/normocapnic air was replaced by hypercapnic air $\left(\mathrm{CO}_{2} 5 \%\right)$, or hypoxic air $\left(\mathrm{O}_{2} 10 \%\right)$ for 5 minutes. We measured the $f R$ (in cycle per $\min , \mathrm{c} \cdot \mathrm{min}^{-1}$ ), the $V_{T}(\mu \mathrm{l})$ normalized as the ratio $V_{T}$ divided by body weight $\left(V_{T} \mu \mathrm{l} . \mathrm{g}^{-1}\right)$, the $V_{E}\left(\mathrm{ml} \cdot \mathrm{g}^{-1} \cdot \mathrm{min}^{-1}\right)$, the number of apneas per minute greater than 2 normal respiratory cycles (apneas $>2 \mathrm{~T}_{\text {TOT }}$ ) expressed as the apnea index (AI), and the IS (variability in duration of respiratory cycles during 5 successive periods) (59). Measurements were performed during (a) control period under air, prior to the challenge, (b) the third minute of the challenge, (c) the fifth minute of the challenge, (d) the postchallenge period (the first 2 minutes after the end of the challenge when normoxia or normocapnia was restored in the plethysmographic chamber), and (e) 10 to 15 minutes later. The inactivation and rescue experiments were analyzed simultaneously; therefore, the same controls (AAV-VN-CT) were used and the values are shown in both graphs. Further details are in Supplemental Methods.

Electrophysiology. All experiments used transverse, rhythmic 600 - to $650-\mu \mathrm{m}-$ thick medullary brain slices obtained from 8- to 12-day-old NesKO, KO, and control mice. Mice were deeply anesthetized with isoflurane (delivered by inhalation) and quickly decapitated at the C3/C4 spinal level (59). Extracellular recordings were obtained with glass suction electrodes positioned on the slice surface in the ventral respiratory group (VRG) near or on top of the preBötC. Intracellular patch-clamp recordings were obtained with a MultiClamp 700B Amplifier (Molecular Devices), using blind-patch technique to VRG neurons in 600 - to $650-\mu \mathrm{m}$ brain stem slice preparations. Further details are in Supplemental Methods.

Histology. Histology was performed as described (23). Briefly, mice were anesthetized with an overdose of pentobarbital perfused with PBS followed by $4 \%$ PFA. Further details are in Supplemental Methods.

Statistics. Data are shown as the mean \pm SEM. GraphPad Prism v5.0 software was used for statistical analyses. Appropriate tests were selected depending on the experimental design as stated in the text. Statistical significance, when reached $(P<0.05$ was considered significant), is stated in the text or figure legends.

Study approval. All animal experiments were approved by the Animal Care and Use Committee at the University of Washington.

\section{Acknowledgments}

This work was supported by a grant from the NW Mitochondrial Guild (to R.D. Palmiter). A. Quintana was a recipient of a Ministerio de Ciencia e Innovación (Spain) (MICINN) Postdoctoral Mobility Program fellowship. The authors thank Jennifer Stone, Raj Kapur, and Elisenda Sanz for their help in this research and Glenda Froelick for assistance with histology.

Received for publication January 17, 2012, and accepted in revised form April 18, 2012.

Address correspondence to: Richard Palmiter, HHMI Box 357370, University of Washington, Seattle, Washington 98195, USA. Phone: 206.543.6064; Fax: 206.543.0858; E-mail: Palmiter@uw.edu.
1. Leigh D. Subacute necrotizing encephalomyelopathy in an infant. J Neurol Neurosurg Psychiatry. 1951;14(3):216-221.

2. Rahman $S$, et al. Leigh syndrome: clinical features and biochemical and DNA abnormalities. Ann Neurol. 1996;39(3):343-351.

3. Arii J, Tanabe Y. Leigh syndrome: serial MR imaging and clinical follow-up. AJNR Am J Neuroradiol. 2000;21(8):1502-1509.

4. Loeffen J, et al. The first nuclear-encoded complex I mutation in a patient with Leigh syndrome. Am J Hum Genet. 1998;63(6):1598-1608.

5. Cavanagh JB, Harding BN. Pathogenic factors underlying the lesions in Leigh's disease. Tissue responses to cellular energy deprivation and their clinico-pathological consequences. Brain. 1994; 117(pt 6):1357-1376.

6. Piao YS, Tang GC, Yang H, Lu DH. Clinico-neuropathological study of a Chinese case of familial adult Leigh syndrome. Neuropathology. 2006; 26(3):218-221.

7. Finsterer J. Leigh and Leigh-like syndrome in chil- dren and adults. Pediatr Neurol. 2008;39(4):223-235.

8. Yasaki E, et al. Characteristics of breathing abnormality in Leigh and its overlap syndromes. Neuropediatrics. 2001;32(6):299-306.

9. Zhang Y, et al. Clinical and molecular survey in 124 Chinese patients with Leigh or Leigh-like syndrome. J Inherit Metab Dis. 2007;30(2):265.

10. Kartikasalwah A, Lh N. Leigh syndrome: MRI findings in two children. Biomed Imaging Interv J. 2010;6(1):e6.

11 . Benit $\mathrm{P}$, et al. Genotyping microsatellite DNA markers at putative disease loci in inbred/multiplex families with respiratory chain complex I deficiency allows rapid identification of a novel nonsense mutation (IVS1nt -1) in the NDUFS4 gene in Leigh syndrome. Hum Genet. 2003;112(5-6):563-566

12. Loeffen JL, et al. Isolated complex I deficiency in children: clinical, biochemical and genetic aspects. Hum Mutat. 2000;15(2):123-134.

13. Petruzzella V, Papa S. Mutations in human nuclear genes encoding for subunits of mitochondrial respiratory complex I: the NDUFS4 gene. Gene.
2002;286(1):149-154.

14. Lebon $S$, et al. Recurrent de novo mitochondrial DNA mutations in respiratory chain deficiency. JMed Genet. 2003;40(12):896-899.

15. Malfatti E, et al. Novel mutations of ND genes in complex I deficiency associated with mitochondrial encephalopathy. Brain. 2007;130(pt 7):1894-1904.

16. Anderson SL, et al. A novel mutation in NDUFS4 causes Leigh syndrome in an Ashkenazi Jewish family. JInherit Metab Dis. 2008;31 suppl 2:S461-S467.

17. Budde SM, et al. Clinical heterogeneity in patients with mutations in the NDUFS4 gene of mitochondrial complex I. J Inherit Metab Dis. 2003;26(8):813-815.

18. Leshinsky-Silver E, et al. NDUFS4 mutations cause Leigh syndrome with predominant brainstem involvement. Mol Genet Metab. 2009;97(3):185-189.

19. Petruzzella V, et al. A nonsense mutation in the NDUFS4 gene encoding the $18 \mathrm{kDa}$ (AQDQ) subunit of complex I abolishes assembly and activity of the complex in a patient with Leigh-like syndrome. Hum Mol Genet. 2001;10(5):529-535.

20. van den Heuvel L, et al. Demonstration of a new 
pathogenic mutation in human complex I deficiency: a 5-bp duplication in the nuclear gene encoding the 18-kD (AQDQ) subunit. Am J Hum Genet. 1998;62(2):262-268.

21. Assouline $Z$, et al. A constant and similar assembly defect of mitochondrial respiratory chain complex I allows rapid identification of NDUFS4 mutations in patients with Leigh syndrome. Biochim Biophys Acta. 2012;1822(6):1062-1069.

22. Kruse SE, Watt WC, Marcinek DJ, Kapur RP, Schenkman KA, Palmiter RD. Mice with mitochondrial complex I deficiency develop a fatal encephalomyopathy. Cell Metab. 2008;7(4):312-320.

23. Quintana A, Kruse SE, Kapur RP, Sanz E, Palmiter RD. Complex I deficiency due to loss of Ndufs 4 in the brain results in progressive encephalopathy resembling Leigh syndrome. Proc Natl Acad Sci US A. 2010;107(24):10996-11001.

24. Telgkamp P, Cao YQ, Basbaum AI, Ramirez JM. Long-term deprivation of substance P in PPT-A mutant mice alters the anoxic response of the isolated respiratory network. J Neurophysiol. 2002; 88(1):206-213

25. Smith JC, Ellenberger HH, Ballanyi K, Richter DW, Feldman JL. Pre-Botzinger complex: a brainstem region that may generate respiratory rhythm in mammals. Science. 1991;254(5032):726-729.

26. Gray PA, Janczewski WA, Mellen N, McCrimmon DR, Feldman JL. Normal breathing requires preBotzinger complex neurokinin-1 receptor-expressing neurons. Nat Neurosci. 2001;4(9):927-930.

27. Hill AA, Garcia AJ 3rd, Zanella S, Upadhyaya R, Ramirez JM. Graded reductions in oxygenation evoke graded reconfiguration of the isolated respiratory network. J Neurophysiol. 2011;105(2):625-639.

28. Solomon IC, Edelman NH, Neubauer JA. Pre-Botzinger complex functions as a central hypoxia chemosensor for respiration in vivo. J Neurophysiol. 2000 83(5):2854-2868

29. Lieske SP, Thoby-Brisson M, Telgkamp P, Ramirez JM. Reconfiguration of the neural network controlling multiple breathing patterns: eupnea, sighs and gasps [see comment]. Nat Neurosci. 2000;3(6):600-607.

30. Corcoran AE, et al. Medullary serotonin neurons and central $\mathrm{CO} 2$ chemoreception. Respir Physiol Neurobiol. 2009;168(1-2):49-58

31. Abbott SB, et al. Photostimulation of retrotrapezoid nucleus phox $2 \mathrm{~b}$-expressing neurons in vivo produces long-lasting activation of breathing in rats. J Neurosci. 2009;29(18):5806-5819.

32. Guyenet PG, Bayliss DA, Stornetta RL, Fortuna MG, Abbott SB, DePuy SD. Retrotrapezoid nucleus, respiratory chemosensitivity and breathing automaticity. Respir Physiol Neurobiol. 2009;168(1-2):59-68.
33. Madisen L, et al. A robust and high-throughput Cre reporting and characterization system for the whole mouse brain. Nat Neurosci. 2010;13(1):133-140.

34. Holstein GR, Friedrich VL Jr, Kang T, Kukielka E, Martinelli GP. Direct projections from the caudal vestibular nuclei to the ventrolateral medulla in the rat. Neuroscience. 2011;175:104-117.

35. Naess K, et al. MtDNA mutations are a common cause of severe disease phenotypes in children with Leigh syndrome. Biochim Biophys Acta. 2009; 1787(5):484-490.

36. Pincus JH. Subacute necrotizing encephalomyelopathy (Leigh's disease): a consideration of clinical features and etiology. Dev Med Child Neurol. 1972;14(1):87-101.

37. Papa S, et al. Mutation in the NDUFS4 gene of complex I abolishes cAMP-dependent activation of the complex in a child with fatal neurological syndrome. FEBS Lett. 2001;489(2-3):259-262.

38. Pronicka E, et al. Compulsory hyperventilation and hypocapnia of patients with Leigh syndrome associated with SURF1 gene mutations as a cause of low serum bicarbonates. J Inherit Metab Dis. 2001; 24(7):707-714.

39. Heymans C, Bouckaert JJ. Sinus caroticus and respiratory reflexes: I. Cerebral blood flow and respiration. Adrenaline apnoea. J Physiol. 1930;69(2):254-266.

40. Solomon IC. Ionotropic excitatory amino acid receptors in pre-Botzinger complex play a modulatory role in hypoxia-induced gasping in vivo. J Appl Physiol. 2004;96(5):1643-1650.

41. Telgkamp P, Ramirez JM. Differential responses of respiratory nuclei to anoxia in rhythmic brain stem slices of mice. J Neurophysiol. 1999;82(5):2163-2170.

42. Curran AK, Rodman JR, Eastwood PR, Henderson KS, Dempsey JA, Smith CA. Ventilatory responses to specific CNS hypoxia in sleeping dogs. J Appl Physiol. 2000;88(5):1840-1852.

43. Dubreuil V et al. A human mutation in Phox 2b causes lack of $\mathrm{CO} 2$ chemosensitivity, fatal central apnea, and specific loss of parafacial neurons. Proc Natl Acad Sci U S A. 2008;105(3):1067-1072.

44. McKay LC, Janczewski WA, Feldman JL. Sleepdisordered breathing after targeted ablation of preBotzinger complex neurons. Nat Neurosci. 2005; 8(9):1142-1144.

45. Sterky FH, et al. Altered dopamine metabolism and increased vulnerability to MPTP in mice with partial deficiency of mitochondrial complex I in dopamine neurons. Hum Mol Genet. 2012; 21(5):1078-1089.

46. Gourine AV, Llaudet E, Dale N, Spyer KM. Release of ATP in the ventral medulla during hypoxia in rats: role in hypoxic ventilatory response. J Neurosci.
2005;25(5):1211-1218.

47. Mironov SL, Langohr K, Haller M, Richter DW. Hypoxia activates ATP-dependent potassium channels in inspiratory neurones of neonatal mice. J Physiol. 1998;509(pt 3):755-766.

48. Miyake A, Yamada K, Kosaka T, Miki T, Seino S, Inagaki N. Disruption of Kir6.2-containing ATPsensitive potassium channels impairs maintenance of hypoxic gasping in mice. Eur J Neurosci. 2007; 25(8):2349-2363.

49. Yates BJ, Bronstein AM. The effects of vestibular system lesions on autonomic regulation: observations, mechanisms, and clinical implications. J Vestib Res. 2005;15(3):119-129.

50. Kumar R, Macey PM, Woo MA, Alger JR, Harper RM. Diffusion tensor imaging demonstrates brainstem and cerebellar abnormalities in congenital central hypoventilation syndrome. Pediatr Res. 2008; 64(3):275-280

51. Krous HF, Chadwick AE, Haas E, Masoumi H, Stanley C. Sudden infant death while awake. Forensic Sci Med Pathol. 2008;4(1):40-46.

52. Harper RM. Sudden infant death syndrome: a failure of compensatory cerebellar mechanisms? Pediatr Res. 2000;48(2):140-142.

53. Greenough G, Sateia M, Fadul CE. Obstructive sleep apnea syndrome in a patient with medulloblastoma. Neuro Oncol. 1999;1(4):289-291.

54. Zheng Y, Umezaki T, Nakazawa K, Miller AD. Role of pre-inspiratory neurons in vestibular and laryngeal reflexes and in swallowing and vomiting. Newrosci Lett. 1997;225(3):161-164.

55. Xu F, Zhuang J, Zhou TR, Gibson T, Frazier DT. Activation of different vestibular subnuclei evokes differential respiratory and pressor responses in the rat. J Physiol. 2002;544(pt 1):211-223.

56. Conrad SC, Nichols NL, Ritucci NA, Dean JB, Putnam RW. Development of chemosensitivity in neurons from the nucleus tractus solitarii (NTS) of neonatal rats. Respir Physiol Neurobiol. 2009; 166(1):4-12.

57. Fadok JP, Dickerson TM, Palmiter RD. Dopamine is necessary for cue-dependent fear conditioning. J Neurosci. 2009;29(36):11089-11097.

58. Bruchas MR, et al. Selective p38alpha MAPK deletion in serotonergic neurons produces stress resilience in models of depression and addiction. Neuron. 2011;71(3):498-511.

59. Viemari JC, et al. Mecp2 deficiency disrupts norepinephrine and respiratory systems in mice. J Neurosci. 2005;25(50):11521-11530.

60. Franklin KB, Paxinos G. The Mouse Brain in Stereotaxic Coordinates. 3rd ed. New York, New York, USA: Academic Press; 2008 